\title{
Implementing an Institutional Repository: The DSpace Experience at MIT
}

\author{
Patsy Baudoin \\ Margret Branschofsky
}

\begin{abstract}
SUMMARY. This paper describes MIT Libraries' experience implementing DSpace, a home-grown open source digital institutional repository, which other institutions may want to introduce as a service to their communities. MIT's introduction of DSpace as an operating service illustrates the many political and organizational considerations that must be addressed to establish and operate institutional repositories. In addition to detailing some of the policies developed and organizational changes undertaken, this article describes the kinds of questions future implementers of DSpace will want to answer. It also outlines the impacts the service has had on the library, on MIT, and on the perception of MIT Libraries within the Institute. [Article copies available for a fee from The Haworth Document Delivery Service: 1-800-HAWORTH. E-mail address: $<$ docdelivery@haworthpress.com> Website: <http://www.HaworthPress.com> (C) 2003 by The Haworth Press, Inc. All rights reserved.]
\end{abstract}

KEYWORDS. Digital libraries, digital institutional repository, digital archive, library policies

Patsy Baudoin, $\mathrm{PhD}$, is Library Consultant for MIT Libraries, 26R Antrim Street, Cambridge, MA 02139 (E-mail: patsybaud@ comcast.net). Margret Branschofsky, MLS, is DSpace User Support Manager, MIT Libraries, Building 14S-M48, 77 Massachusetts Avenue, Cambridge, MA 02139 (E-mail: margretb@mit.edu).

[Haworth co-indexing entry note]: "Implementing an Institutional Repository: The DSpace Experience at MIT." Baudoin, Patsy, and Margret Branschofsky. Co-published simultaneously in Science \& Technology Libraries (The Haworth Information Press, an imprint of The Haworth Press, Inc.) Vol. 24, No. 1/2, 2003, pp. 31-45; and: Innovations in Science and Technology Libraries (ed: William Miller, and Rita M. Pellen) The Haworth Information Press, an imprint of The Haworth Press, Inc., 2004, pp. 31-45. Single or multiple copies of this article are available for a fee from The Haworth Document Delivery Service [1-800-HAWORTH, 9:00 a.m. - 5:00 p.m. (EST). E-mail address: docdelivery@haworthpress.com].

http://www.haworthpress.com/web/STL

(C) 2003 by The Haworth Press, Inc. All rights reserved.

Digital Object Identifier: 10.1300/J122v24n01_04 


\section{THE BIRTH OF AN IDEA}

The idea to create a digital institutional repository ${ }^{1}$ at MIT emerged from conversations between the director of the MIT Libraries, Ann J. Wolpert, and members of the Institute's faculty. She began noticing a need: she quotes one faculty member as lamenting in a joking fashion that his "entire life's work is in [his] email." This message, coinciding with the shifting trends of electronic scholarly publishing as well as the community's increasing requests for on-demand document delivery, set Director Wolpert on the track that led to DSpace. Because university communities rely on their libraries to provide continuing access to research and scholarship, Wolpert and the Assistant Director for Technology, Planning, and Administration at the MIT Libraries positioned the Libraries to devise a solution to the problem of storing and retrieving MIT's intellectual work over the long term. Her on-going conversations with an MIT computer scientist eventually led to a meeting with Hewlett-Packard Labs, with whom the MIT Libraries signed a co-development contract in March 2000.

From the outset, the plan was to create an infrastructure for storing the digitally born, intellectual output of the MIT community and to make it accessible over the long term to the broadest possible readership. How might one store and manage the intellectual output of the MIT community so that it won't sink, forever lost, into the quicksand of software and hardware obsolescence?

\section{A BRIEF CHRONOLOGY}

From contract (March 2000) to launch (November 2002) the DSpace development team was created, the scope of the project was defined, and the system was designed, prototyped, developed, re-designed, tested, and finally launched. By the fall of 2001, a Libraries transition team was in place to determine the smoothest course for adding DSpace services to the MIT Libraries' existing services. By July 2002, two business strategists had accomplished their year-long assessment of how to sustain DSpace into the future financially. In the meantime, DSpace's early adopters came on board in March 2002 and worked with the DSpace team through September 2002, when their content became publicly available, to test the whole system and the processes that needed tweaking. DSpace was officially launched on the same date its source code was released under Open Source BSD license: November 4, 2002. 


\section{WHAT IS DSpace?}

DSpace means different things to different people and constituencies. Sometimes it refers to technology-for DSpace is a computer application. DSpace followed the librarian's inclination to create a system that would be as easy as possible to implement and use, rather than push strictly in the direction of digital library research from which a more flexible system might have emerged. DSpace, therefore, was designed as an open source application that institutions and organizations could run with relatively few resources. The intention to support interoperability (with DSpace implementers at other institutions, for example) led to the adoption of the Open Archives Initiative Protocol for Metadata Harvesting (OAI-PMH). ${ }^{2}$ The OAI Registry includes DSpace, making its Dublin-Core-formatted metadata available to compatible harvesting code. In addition, DSpace chose to implement CNRI Handles $^{3}$ as the persistent identifiers associated with each item to ensure that the system will be able to locate and retrieve documents in the distant future. DSpace was also designed with a batch load submission feature to ease the loading of existing collections and cut costs.

DSpace the technology, then, is the software-the open source computer application that drives and manages submission, storage, and retrieval processes. But DSpace has non-technological aspects of importance, which are the subject of this article.

- DSpace, the program. Conceived in the late 1990s, the initial project took formal flight with the March 2000 signing of a contract between Hewlett-Packard Laboratories and the MIT Libraries. The research and development program is now in its third year and has become a central feature of the MIT Libraries' research agenda.

- DSpace, the service. Management and preservation services are provided for MIT faculty and communities while access to the intellectual output of the MIT community is offered by the MIT Libraries to the MIT community and the world beyond it.

- DSpace is also, finally, the name of the open source software platform on which MIT's institutional repository service is built.

There are aspects of DSpace with which users will need to acquaint themselves: the set of services, the policies, the submission process, issues of access, and so on. Offering DSpace services at MIT required: 
- Planning and developing a set of services that reflect MIT and Libraries' policies

- Finding and working with early adopters

- Hiring staff

- Advocating and getting the word out

- Training the Libraries' staff and the DSpace communities

- Rolling the services out to the MIT community and to the public at large

- Financially sustaining the repository

- Managing and preserving the data so it remains retrievable

For each of these activities, DSpace paid special attention to its major stakeholders and supporters, both regarding the impact the new services might have on each, and with respect to the role each might play in promoting, supporting, and, of course, using DSpace. The constituencies that DSpace kept in focus were:

- The MIT faculty, whose scholarship and research DSpace will usher into the future

- The staff of the MIT libraries, who are responsible for deploying, running, and maintaining the DSpace system and services into the future

- The MIT administration-long-term planners and decision-makers-in light of the Institution's intellectual and financial commitment to DSpace

- Members of the MIT community, whose intellectual and political influence focus attention and develop momentum in support of DSpace

- The early adopters-and their eventual successors, the so-called fast followers

\section{RAISING AWARENESS AND ADVOCATING}

A project of the magnitude of DSpace must market itself and garner the support that matters to its survival; that support must come from key members of the community and spread organically, by word-of-mouth. Opinion leaders, key administrators, and respected members of the faculty must be courted and persuaded; their conversation about the project will create buzz and their endorsement will promote credibility. At 
MIT, word-of-mouth, a most infectious means of disseminating excitement, continues to prove very effective.

Though informal excitement spreads like wild fire, the fire had to be lit and stoked by planned, more formal interventions: the idea of DSpace had to be made to capture the imagination of the faculty-and the implementation had to offer the solution to problems some may not even have known they had. The DSpace team introduced DSpace to members of the MIT community both programmatically and informally. In addition to their individual, enthusiastic, everyday conversations, the team prepared presentations for small groups which had expressed curiosity, and created forums for discussion of what DSpace would be and how it would help the MIT community and its various parties. The Director presented the program to her colleagues on campus and helped solidify arrangements with early adopters. A free DSpace-Announce electronic mailing regularly reached subscribers, reporting on the project's news and progress. The Libraries' public relations office sent press releases about DSpace's milestones to the news media outside of MIT. Their pieces are collected at http://dspace. org/news/dspace-news.html. The word was getting out.

\section{THE DSpace TEAM}

To keep the project moving ahead, some organizational changes took place. The project's team was formalized: a project manager from HP Labs joined several HP and MIT software engineers and MIT librarians. One of the librarians served as liaison to the faculty. The team also included two business strategists who helped in the process of moving the project into the daily operations of the Libraries in addition to recommending funding models for DSpace's long-term sustainability.

The Libraries' Director strategically invited specific faculty members to sit on the newly formed DSpace Faculty Advisory Board to help guide and support the project and its working team members. Their familiarity with the concept raised healthy discussion among their peers and continues to proffer invaluable advice as the project moves forward.

As word about DSpace circulated and the team took shape, a flood of questions began coming in. What content would DSpace house? How much of it could be submitted? Who would submit content? Could one remove a submission later? What about this digital format or that? It 
was time to attend to the many policy questions both the DSpace team and the DSpace buzz generated.

\section{DEVISING SOUND POLICIES}

One of the most complex aspects of introducing an institutional repository into the varied set of library services is setting the policies. MIT's DSpace policy development process, which continues today and will continue into the future, reflects the complexity of the issues at hand. It also reveals the MIT community's constantly evolving awareness of DSpace's potential.

Cognizant of the wisdom that policies ought to shape software development and not the reverse, DSpace's technical team often sought policy decisions before moving forward with their coding. There was no shortage of decisions to be made to move ahead with the software's development. Some policy decisions, driven by the MIT community's needs and culture, affected how the DSpace software and infrastructure can and will be used. Other policy decisions reflected the Institute's and the Libraries' missions and commitments-and will have to be shaped on an individual institutional basis by other DSpace implementing institutions. Philosophically, MIT followed a "reasonable request" principle in fashioning policies: while not being able to subsidize all requests, the goal was to remain, above all, very service-oriented. In the MIT experience, input from various parts of the MIT community influenced the fashioning of policy.

Among the early policy decisions the DSpace team discussed and settled on were those involving the scope and boundaries of the DSpace service the MIT Libraries would offer-in large measure to get better definition of what it was the team was creating. Most of these early policy decisions resulted from philosophical commitments and the Libraries' mission-e.g., open source software, free access wherever possible to anyone browsing the web, etc. As the project evolved, the need to create policy emerged from several corners of the MIT community. Feedback was a crucial conduit in making sure that DSpace would suit MIT's culture. The Institute's administrators and staff, the faculty, and the staff of the MIT Libraries variously contributed to this on-going feedback loop:

- Inquiring faculty members raised a great number of very important questions. Several faculty members, for example, asked about re- 
moving old versions of re-written articles, which prompted thinking through what might indeed be withdrawn from DSpace, if anything at all, and under what circumstances. Two surveys were also delivered to the faculty, each with a dual purpose: to request the faculty's input and to inform it of what was afoot.

- The Libraries' administrators and staff, in the course of introducing the DSpace concept to the MIT community, helped the DSpace team understand what sorts of things needed to be explained in greater depth, what sorts of trainings needed to be planned, and how efforts had to be sustained.

- In meetings with non-DSpace-team Libraries' staff, the DSpace team learned more about what organizational changes would be needed to manage and maintain the DSpace service once it became part of the MIT Libraries' services.

Drawing up policy can be difficult because it often affects many constituencies. Librarians typically want to maintain the scholarly record as a part of their service mission. Faculty members may hesitate to submit their research if the commitment is irreversible. Administrators may want to support cost-cutting policies wherever possible, sometimes running afoul of either operations or faculty needs. MIT's experience is that compromise is quite attainable-and makes the DSpace service all the stronger following careful, in-depth debate.

Setting policy also drives further work for the DSpace team and for the submitting communities. Here is one example. After a great deal of discussion, the DSpace team decided as a matter of policy that each community would be responsible to enter metadata for the items it deposits into DSpace. Establishing this policy meant that developers needed to create very intuitive web interfaces to accept the submitter's input. It also meant that submitters in each and every community would need to be trained to enter appropriate metadata so that the quality of the metadata would be acceptable. Recognizing that an easy-to-use submission process would reduce barriers to faculty participation, the DSpace team's policy decision led to a minimum requirement for metadata consisting of only three fields. Requiring only three fields, but encouraging many more, it is hoped, will have the extra effect of lowering the barrier to entry for submitters with insufficient time on their hands to attend to the fuller process.

Some policies reflect current knowledge about available technologies and will need to be refined as technologies evolve and new ones replace existing ones. Owing to the varieties of formats used by MIT's 
faculty in its research, for example, the DSpace team realized it would have to accept research in many different digital formats. But because the means to preserve some of these formats are still under investigation by researchers in the field of digital preservation, the DSpace service could not promise to preserve all formats with equal success. To address these differences, the team devised several levels of service promise, which DSpace describes in greater detail at http://dspace.mit.edu.

The process of refining policies continues as new questions arise. Having expressed some disparate policies early on, for example, the DSpace team later created a policy statement for submitters, to assure that everyone understands the terms of the Libraries' commitment and responsibilities as well as those of the submitting community. To monitor the quality of policies, to keep them current and in tune with MIT's culture, and to enforce them, the MIT Libraries created The DSpace@MIT Policy Committee, even as DSpace was rolled out to the public. This committee is charged "with ongoing responsibility for advising the Associate Director for Technology on all aspects of policy related to deployment of the DSpace system at MIT." As more communities use the system, new policy issues arise and more policies are defined. At any given time, DSpace current policies are readily available at http://dspace.org/mit/policies/index.html. To read them is to get a sense of the breadth and depth of what a DSpace implementation requires to assure that services be rolled out smoothly and legally.

\section{THE SUSTAINABILITY IMPERATIVE}

As the development of DSpace progressed and before it could actually be introduced into the broader set of MIT Libraries' services, the question of financial sustainability gnawed. It was one of the most persistently difficult questions and it emerged at the outset: assuming this repository could indeed be built, how would the Libraries at MIT sustain it? Where would the monies come from to live up to the promise of data persistence and retrievability over the long haul?

Since the idea of an institutional repository was a new one for which a financial model did not yet exist, MIT Libraries decided to apply for independent funding to do research in this area. The Andrew W. Mellon Foundation granted the MIT Libraries monies to add to the DSpace team two business strategists, whose charge it was to study the problem and devise a sustainable financial plan. Their work resulted in the July 
2002 publication of MIT Libraries' DSpace Business Plan Project Report to the Andrew W. Mellon Foundation. ${ }^{4}$

The importance of planning for the financial support of this sort of endeavor cannot be underestimated. In trying to identify funding sources, several overarching concerns surfaced:

- Topping the list is the open-ended, long-term nature of the project. What would it cost to run, support, maintain, and upgrade DSpace services over many decades?

- Capturing the costs of data preservation, specifically, remains one of the biggest unknowns and least measurable items, in large part because research has yet to be undertaken on the feasibility and cost associated with large-sized data migrations.

- Although storage is not the expensive proposition it used to be, storage must be taken far more seriously when one considers the size of some of the proposed deposits into DSpace. A recent inquiry came to the DSpace team regarding depositing a single, 30-terabyte dataset, for example. Managing the storage capacity for datasets of this size requires careful financial planning. ${ }^{5}$

- Forecasting staffing is also essential since it can quickly become a major expense.

- Finally, introducing DSpace into a library environment will strain already typically allocated funds. Whatever the cost models and financing plans, creative approaches will always be welcomed in tight economic times.

In working on their plan, the business strategists raised several possible configurations for the on-going funding of a service such as DSpace. They stayed attuned to the assumptions that the DSpace team was making-in the process, unearthing issues that would require further policy guidelines and attention.

\section{THE DSpace SET OF SERVICES}

One of the business analysis' outcomes was that it helped define both the Core Services, which the Libraries would make available free of charge; and the Premium Services, for which the Libraries could charge fees. As DSpace grows, further attention will be paid to the Premium Services, which might include accommodating such extraordinary resource demands as extra storage space and customizing services for 
specialized needs. Premium Services will be introduced in a controlled fashion so as not to overtax the Libraries' resources. In the meantime the DSpace team settled on a two-part Core Service to address a broad base of needs and offer basic support and functionality as soon as would be possible. At launch, the Core Service included:

- Managed Services: Broadly speaking, these comprise storage, management, retrieval, and differing levels of preservation (including the requisite back-up, recovery, and general systems monitoring and maintenance).

- End-User Services: These include user support and training in all aspects of the community set-up process, end user support services, and system management support services.

Services beyond these two broad Core Services fall into the category of Premium Services and will be offered on a fee-for-service basis. This category includes digitization of print materials, file conversion, and metadata services. One impact DSpace has had on MIT Libraries is the recent establishment of a metadata unit to provide consultation and metadata creation services to DSpace communities. More information about current DSpace services offered at MIT can be obtained at http:// dspace.org/mit/services.html or please refer to MIT Libraries' DSpace Business Plan Project Report to the Andrew W. Mellon Foundation.

DSpace services exist in concert with policies that sustain them; planning these services and setting policies supported and refracted each other. Creating a model for such a set of services turns out to be an iterative process that needs to begin in the early phases of project planning. The MIT DSpace services evolved throughout as the team learned more about the faculty's needs and the types of research materials faculty members produced. Some of the DSpace team's early expectations-that submissions, for example, would predominantly consist of text materials-were mistaken. The team learned directly from the faculty that it wanted DSpace to manage datasets, images, videos, and other types of communication vehicles, in a variety of digital formats. The DSpace service plan had to reflect that.

\section{DSpace's EARLY ADOPTERS}

The DSpace team formally and programmatically gathered feedback from MIT communities regarding how the DSpace system worked and 
how it met the needs of its users. DSpace's five early adopter communities played particularly significant roles: they tested all aspects and features of the DSpace system's submission processes. They provided invaluable feedback on all of the phases and processes in which they took part.

To recruit early adopters, it was valuable to remind prospects of what early participation would bring them. Early adopters benefited from the visibility that comes with blazing new trails for DSpace, a new, exciting campus-wide technology venture. Their names figured in promotional and publicity materials. They had a hand in tailoring the software and user interfaces. They got to showcase their research ahead of later entrants and reached worldwide audiences promptly. They also got special and prompt attention in solving problems.

Early adopters at MIT represented a cross-section of the Institutegroups of different sizes, submitting varied types of research, using several formats, whose disciplinary cultures and practices vary as well. They are:

- The Center for Technology, Planning and Industrial Development (CTPID)

- The Department of Ocean Engineering

- The Laboratory for Information and Decision Systems (LIDS)

- The Sloan School of Management

- The MIT Press

The DSpace Faculty Liaison also brought into the process five librarians, each a subject specialist with previously established ties to each of the Institute's DSpace early adopter communities, to help coordinate early adopters' efforts.

The team's Faculty Liaison helped early adopters define their communities, which involved, among other things:

- Establishing a list of who plays what role in the submission and review processes.

- Guiding individual communities to make policy decisions affecting the submission and access policies governing their collections. These decisions were made at the highest level of the community, often after several meetings with the Faculty Liaison.

- Identifying the DSpace Coordinator, who stands as the liaison to the DSpace team, and works directly with the team's Faculty Liai- 
son to manage these various steps in setting up the community to get it going, and coordinate the Community's policy setting.

DSpace support includes helping each community establish and name its collections as well as create and test its workflows for submission and review; providing guidance in creating metadata (preferably using existing controlled vocabularies to standardize metadata input); assisting with customizing individual community portal pages; and, finally, helping early adopter communities load and submit their content.

\section{THE TRANSITION}

In the fall of 2001, as the DSpace project was moving ahead on all fronts, it was time for the MIT Libraries to address the introduction of DSpace services into the Libraries' daily operations. A Transition Team was convened in late 2001 to begin the process of ushering DSpace services into the broader set of increasingly electronic services the MIT Libraries provides its patrons. The team was made up of librarians from each of MIT's individual libraries as well as representatives from technology, technical services, and public services areas. The transition team sought to assess the impact this new service would have on the Libraries' staff: what staff would be needed, how much time would staff need to allocate to DSpace work, etc. The results of this impact analysis can be read in The Report of the DSpace Transition Planning Group to the MIT Libraries Steering Committee.

To bring full awareness of DSpace to the staff of the Libraries the transition team recommended a communications plan and a staff training plan. A FAQ, geared to librarians, was created to provide answers to some of the most frequently asked questions. It includes information among other things regarding policies; services; DSpace's relationship to such kindred campus-wide projects as OpenCourseWare (http://ocw. mit.edu/index.html); and whom to contact with questions. The DSpace team also planned formal trainings and a less formal brown-bag lunch to complement the occasional updates the Libraries staff heard about DSpace at periodic all-staff meetings.

The transition team also recommended some organizational changes that MIT Libraries implemented:

- It recommended putting in place the DSpace@MIT Policy Committee to create, refine, monitor, and enforce DSpace policies. 
- It recommended putting in place the DSpace Advisory Committee.

- It recommended the creation of two staff positions, namely the DSpace Systems Manager and the DSpace User Support Manager.

Throughout the process, the librarian members of the DSpace team wore many hats, including translating and serving as ambassadors between the rest of the team and everyone else within and without the Libraries. This role needs to be maintained well after DSpace is implemented in order to make sure that non-technical library staff may continue to contribute to the continued evolution of user-centered DSpace services.

\section{IMPACTS}

DSpace itself introduces into both the institution and the library hosting DSpace new ways of thinking about well-ensconced workflows and policies. Faculty members, for example, must participate actively in making sure their research doesn't fall prey to the obsolescence of specific information technologies; they must actively seek the management help of DSpace, under whose stewardship the research will be brought forward as technologies do change or disappear. At the same time, collection managers increasingly need to be thinking of their work no longer, for example, at the level of journals and books only, but also at the level of individual articles or datasets. The introduction of DSpace, in other words, changes not only the way we think about the lifecycle of scholarly research, but also the operating definitions of units of the scholarly enterprise.

Implementation of the DSpace communications plan proved to be very successful in that it led to widespread knowledge of the project both within and outside of MIT. Early on the development team initiated communication with those involved in related digital projects on the MIT campus. Course management systems such as Stellar, ${ }^{6}$ SloanSpace, ${ }^{7}$ and Command ${ }^{8}$ are now interested in DSpace as tools for managing and preserving collections of reusable course materials. MIT's OpenCourseWare (OCW $)^{9}$ initiative is working with DSpace to store its superceded course web sites. OCW has also contracted with the Libraries' newly established Metadata Unit to provide it with metadata. SloanSpace is developing an authoring system that will feed its results into DSpace. And DSpace is one of two repository systems for which an Open Service Interface Definition is being planned through the Open 
Knowledge Initiative (OKI). ${ }^{10}$ As a result of connections like these, DSpace is increasingly seen as an active player in developing technical infrastructure at MIT, as well as a source of expertise in the areas of metadata and content management. DSpace representatives are now invited to participate in campus technology activities such as last year's Technology Fair, and the DSpace faculty liaison has become a member of the Educational Technology Partners group composed of staff from the many technology initiatives on campus.

The Libraries have also become more visible in non-technical corridors of the Institute. DSpace has brought interesting intellectual property issues to the attention of faculty, administrators, and MIT's IP counsel. Grant proposal requirements asking researchers to provide a plan for sharing and preserving their research results have led to the inclusion of DSpace information in grant proposals, with involvement of the Office for Sponsored Research. The introduction of electronic theses into DSpace will have a large impact on MIT's thesis submission process and will involve the office of the Dean for Graduate Students.

Since the initial HP grant award in 2002, MIT Libraries has been the recipient of six further grants from four different granting bodies, all to support activities related to DSpace. A newly created library section, the Digital Library Research Group, is composed of staff assigned to carry out these activities. Although the sum of the awards does not rival those of most MIT departments and research centers, it does represent a significant change in the Libraries' status at MIT. The Libraries is no longer perceived as a support service that only takes money out of the general budget, but is seen rather as a contributor to research, which brings money into the Institute. This coupled with the large amount of publicity DSpace has garnered outside of the University has brought a measure of on-campus recognition to the Libraries.

\section{FUTURE}

From the beginning, DSpace was planned as an Open Source project whose software would be freely available to other institutions. Several institutions worldwide already have a DSpace system in production. Through grants from several funding bodies, a pilot federation of interested institutions was formed to test the transferability of the software into other environments and also to explore the possibilities for the sharing of both technical and other resources to further the aims of digital 
preservation and scholarly communication. It is hoped that work to enhance the system will be produced by many DSpace implementers. (See the DSpace Federation website http://www.dspace.org, for updates.)

As DSpace at MIT approaches the end of its first year of production, its staff is actively marketing the service to prospective contributing communities on campus. The System Manager is purchasing more storage space to accommodate the collections that are being readied for a data release this September. Policy issues continue to emerge. The business model is being tested. Worldwide attention is increasing. DSpace progress can be monitored at http://dspace.mit.edu and http://www. dspace.org.

\section{NOTES}

1. The MIT Libraries espouses SPARC's use of the term "institutional repository" to denote "digital collections capturing and preserving the intellectual output of a single university or a multiple institution community of colleges and universities." (http://www.arl.org/sparc/IR/IR_Guide.html\#repository).

2. Open Archives Initiative Protocol for Metadata Harvesting (OAI-PMH), http:// www.openarchives.org/OAI/openarchivesprotocol.htm.

3. Information about the Handle System ${ }^{\circledR}$ is available at http://www.handle.net.

4. http://libraries.mit.edu/dspace-mit/mit/plan.html.

5. Stephen Chapman analyzes the storage costs of long-term, digital preservation in "Counting the Costs of Digital Preservation: Is Repository Storage Affordable?" in Journal of Digital Information, volume 4, issue 2, http://jodi.ecs.soton.ac.uk/Articles/ v04/i02/Chapman/.

6. http://stellar.mit.edu/.

7. http://sloanleadership.mit.edu/ssfaq.php.

8. http://command.mit.edu.

9. http://ocw.mit.edu.

10. http://www.mit.edu/afs/athena/project/oki/specs/index.html. 